\title{
Enhanced Production of Runaway Electrons during a Disruptive Termination of Discharges Heated with Lower Hybrid Power in the Frascati Tokamak Upgrade
}

\author{
J. R. Martín-Solís, ${ }^{1, *}$ B. Esposito, ${ }^{2}$ R. Sánchez, ${ }^{3}$ F. M. Poli, ${ }^{4}$ and L. Panaccione ${ }^{2}$ \\ ${ }^{1}$ Departamento de Física, Universidad Carlos III de Madrid, 28911 Leganés, Madrid, Spain \\ ${ }^{2}$ Associazione Euratom-ENEA sulla Fusione, CR Frascati, CP 65, 00044-Frascati, Roma, Italy \\ ${ }^{3}$ Fusion Energy Division, Oak Ridge National Laboratory, Oak Ridge, Tennessee 37831, USA \\ ${ }^{4}$ Centre de Recherches en Physique des Plasmas, EPFL, Lausanne, Switzerland
}

(Received 26 July 2006; published 18 October 2006)

\begin{abstract}
We report on the observation of a large production of runaway electrons during a disruptive termination of discharges heated with lower-hybrid waves at the Frascati Tokamak Upgrade. The runaway current plateaus, which can carry up to $80 \%$ of the predisruptive current, are observed more often than in normal Ohmic disruptions. The largest runaway currents correspond to the slowest plasma current decay rates. This trend is opposite to what is observed in most tokamaks. We attribute this anomalous behavior to the acceleration of the preexistent wave-resonant suprathermal electrons during the disruption decay phase. These results could be relevant for the operation of the ITER tokamak whenever a sizeable amount of lower-hybrid power is made available.
\end{abstract}

DOI: 10.1103/PhysRevLett.97.165002

The high electric fields induced during the current quench phase of a tokamak disruption can generate a large number of runaway electrons with energies as high as hundreds of $\mathrm{MeV}$ [1]. Experimentally, runaway current plateaus of a few mega-amps have indeed been reported in large tokamaks like JET or JT-60U [2,3]. In consequence, there is a great concern about the damage that these energetic electrons might cause if they impact on the first wall structures, which might be critical for larger devices such as the ITER tokamak.

It is widely accepted that runaway electrons are generated via two mechanisms. Primary (or Dreicer) generation happens via a diffusion process in velocity space [4]. Secondary (or avalanche) generation occurs when runaway electrons kick thermal electrons past the critical energy and turn them into runaway electrons [5]. In an ITER disruption, it is been predicted that avalanching would dominate and turn as much as two-thirds of the predisruption current into the runaway current [6]. The theoretical understanding of these processes suggests that the runaway production rate is correlated with the current quench rate [7]. Recent JET disruption data are consistent with this view: the runaway current apparently scales linearly with the plasma current derivative as well as with the predisruptive plasma current [8]. This correlation may provide a knob to control the runaway production in case of a disruption.

In this Letter we report on the first experimental observations that suggest that this correlation may not hold in general. Our results have been obtained in the current decay phase of disruptions taking place at the Frascati Tokamak Upgrade (FTU) during the plasma current flattop of lower-hybrid (LH) wave heated limiter discharges. FTU [9] is a tokamak with a circular cross section (major radius $R_{0}=0.935 \mathrm{~m}$, minor radius $a=0.3 \mathrm{~m}$ ) with a high toroidal magnetic field (up to $8 \mathrm{~T}$ ) and $\mathrm{LH}$ power $(f=8 \mathrm{GHz})$
PACS numbers: 52.55.Fa, 52.35.Py, 52.50.Sw, 52.55.Pi

up to $2 \mathrm{MW}$. In the past, the analysis of a large number of FTU Ohmic hydrogen and deuterium disruptions showed that the case was rare in which a runaway current plateau followed the disruption current quench (less than $5 \%$ of cases) [10]. In contrast, runaway current plateaus are observed more often in disruptions happening during $\mathrm{LH}$ injection. The fraction of the predisruption plasma current converted to the runaway current is very large, often exceeding $50 \%$ and reaching values as high as $80 \%$ of the predisruption current. But more surprisingly, the runaway current plateau does not increase with the plasma current derivative. Instead, it becomes smaller the faster the current decay rate is. We will argue that this anomalous behavior, in apparent contradiction with both Dreicer and avalanche generation, is due to the acceleration of the suprathermal electrons (previously created by the LH waves) during the disruption current quench. These findings could be important for the operation of ITER once the significant amount of lower-hybrid power planned for future upgrades becomes available.

To facilitate the understanding of runaway measurements during a disruption, we first describe how $\mathrm{MeV}$ runaway electrons are detected in FTU disruption-free LH discharges. Runaway electrons can be produced in the postheating phase through the acceleration of the fast LH resonant electrons [11]. An example is provided in Fig. 1 for deuterium discharge No. $18670\left(I_{p} \simeq 350 \mathrm{kA}\right.$; $P_{\mathrm{LH}} \simeq 0.9 \mathrm{MW}$; parallel wave refractive index $N_{\|}=1.8$ ). The LH waves are injected during the plasma current flattop of the discharge $(\sim 0.5-1.2 \mathrm{~s})$ giving rise to a population of resonant fast electrons via Landau damping (with energy $E_{\text {res }} \simeq 105 \mathrm{keV}$ ). These electrons are detected by a fast electron bremsstrahlung (FEB) camera with 17 horizontal lines of sight that measures, in the energy range 20$200 \mathrm{keV}$, the bremsstrahlung $\mathrm{x}$-ray emission from the plasma perpendicular to the magnetic field [12]. This is 


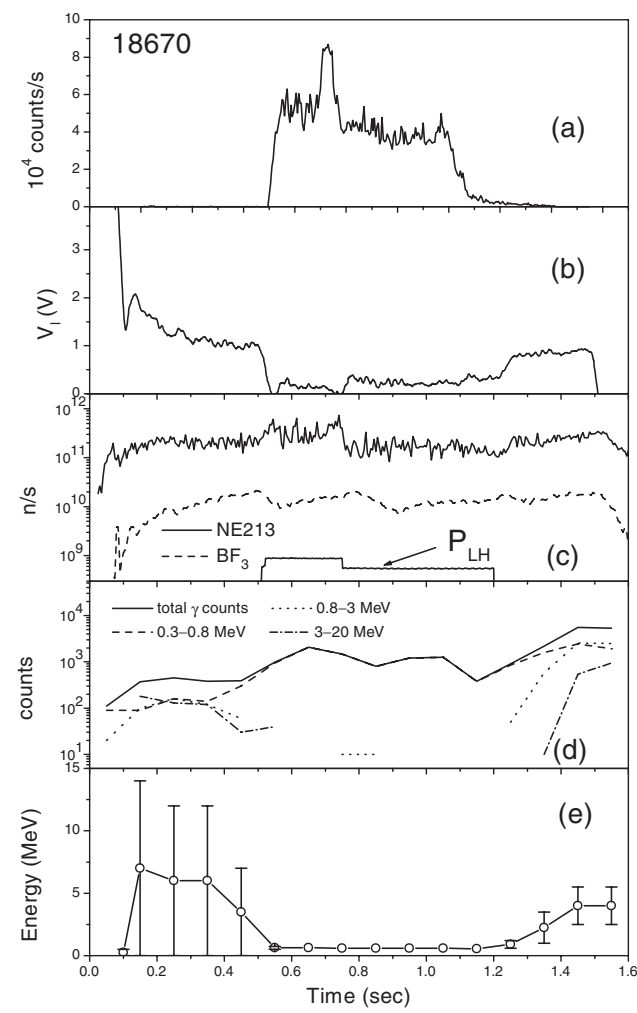

FIG. 1. Time traces of line integrated FEB emission (central chord), loop voltage, $B F_{3}$ and NE213 scintillator, photon counts detected by the $\gamma$-ray system, and maximum $\gamma$ energy for the $\mathrm{LH}$ deuterium discharge No. 18670.

illustrated in trace (a) of Fig. 1 (central chord, channel 9). The large drop of the loop voltage $[\sim 80 \%-90 \%$ of the preheating $V_{l}$ value observed in trace (b)] is due to the replacement of the resistive plasma current by that carried by the LH suprathermal electrons. Frames (c)-(e) of Fig. 1 illustrate the runaway measurements. A direct indication of runaway presence is obtained by comparing the data collected by a set of $B F_{3}$ counters that detect only neutrons (due to DD fusion reactions and/or to photoneutron reactions [13]) with the data collected by a NE213 scintillator sensitive to both neutrons and $\gamma$ rays [see trace (c)]. During discharges with negligible runaway population, the two traces overlap. But when runaway electrons are present, the NE213 signal shows an excess of $\gamma$-ray events and no longer equals the $B F_{3}$ signal [12]. For instance, the large difference observed in Fig. 1(c) during the first $500 \mathrm{~ms}$ of the discharge is due to the runaway electrons created during the current ramp-up (approximately the first $200 \mathrm{~ms}$ ), when the electric field is high and the plasma density is low $\left(\leq 2 \times 10^{19} \mathrm{~m}^{-3}\right)$. In contrast, during the current flattop the electric field has decreased and the density is higher so no more runaway electrons are produced, but the ones produced previously can still stay confined for the rest of the discharge [12]. More information about the runaway population is obtained with a $\gamma$-ray spectrometer [14] that views the plasma on the equatorial plane and collects energy spectra in the range $0.3-23 \mathrm{MeV}$ with a time resolution of $0.1 \mathrm{~s}$. The $\gamma$-ray measurements are typically thick target bremsstrahlung spectra produced by runaway electrons that hit the limiter and the vessel structures. Figure 1(d) shows the time trace of the photon counts (total number and distribution in several energy ranges). It is clear that the low energy range emission $(0.3-0.8 \mathrm{MeV})$ increases during the $\mathrm{LH}$ injection phase due to the creation of resonant suprathermal electrons. This population is responsible for the observed difference between the NE213 and the $B F_{3}$ signals during this phase. Note that the $\mathrm{MeV}$ runaway population $(0.8-20 \mathrm{MeV})$ that was created at startup is suppressed by the large electric field drop that takes place during LH current drive. This is illustrated in trace (e), which shows the runaway maximum energy estimated as the largest $\gamma$-ray energy detected [12]. This energy increases during ramp-up up to $\sim 6 \mathrm{MeV}$ but experiences a large drop during the LH phase, when the loop voltage is reduced almost to zero. But note also that the runaway maximum energy and the $\mathrm{MeV}$-range $\gamma$-ray count rate signals recover their preheating level later [see traces (d)-(e)], a fact that will be of central importance to the understanding of the LH-disruption data. This recovery is not observed in discharges heated with electron cyclotron power [15], which also suppresses the runaway population during the heating phase, although the drop of the electric field is noticeably smaller than in the LH case. The explanation for the recovery must thus be sought in the population of suprathermal electrons that was created via Landau damping of LH waves. Once the heating phase is over, these electrons are accelerated by the restored electric field and they replenish the $\mathrm{MeV}$ runaway ranks. This interpretation is supported by simulations which show that the energy that these suprathermal electrons (approximately hundreds of $\mathrm{keV}$ ) may gain in the post-LH plasma conditions is consistent with the MeV-range energies measured after the LH phase [11]. The same simulations also show that the post-LH plasma conditions do not allow for any significant Dreicer or avalanche generation.

We discuss now the observations referring to disruptions taking place within the flattop of discharges in which LH was being applied. These deuterium discharges belong to the 2001 experimental campaign and their typical parameters are the following: plasma current $I_{p} \sim 0.3$ or $0.5 \mathrm{MA}$, toroidal magnetic field $B_{0}=5-7 \mathrm{~T}$, and central line averaged density $\bar{n}_{e}=(4-12) \times 10^{19} \mathrm{~m}^{-3}$. The wave parallel refractive index $N_{\|}=1.5$ or 1.8 (resonant electron energy $\sim 175$ and $100 \mathrm{keV}$, respectively), and the input power varied in a range from 0.3 to $2 \mathrm{MW}$. In contrast to the behavior observed in Ohmic disruptions, when runaway current plateaus were observed in only $\sim 2 \%$ of the cases, they were found in $\sim 15 \%$ of the LH disruptions. A typical LH disruption is shown in Fig. 2 [discharge No. 19989; predisruption plasma current $I_{p} \simeq 0.52 \mathrm{MA} ; B_{0} \simeq 7.1 \mathrm{~T}$; $\left.\bar{n}_{e} \simeq 5 \times 10^{19} \mathrm{~m}^{-3} ; P_{\mathrm{LH}} \simeq 1.7 \mathrm{MW} ; N_{\|}=1.8\right]$. A runaway current plateau $\left(I_{r} \simeq 0.3 \mathrm{MA}\right)$ is observed at the end of the disruption current quench. The dashed line in 
Fig. 2(a) represents the loop voltage signal during the disruption. The usual initial negative loop voltage spike [16] is followed by a positive spike associated with the large electric field induced by the plasma cooling during the thermal quench which appears to be correlated to the plasma current derivative. The neutron measurements reveal the formation of a large runaway population. The increase in the photoneutron emission [Fig. 2(b), solid line] is noticeable and lasts for the whole runaway current plateau. The increase in the scintillator signal [Fig. 2(b), dashed line] is even more pronounced and usually saturates. In contrast, in disruptions without runaway current plateau, the neutron signals typically fall at the time of the disruption or, at most, show a peak at the beginning of the current decay associated with a small runaway production.

We estimate the runaway energy by assuming that runaway electrons are produced at the start of the current quench,

$$
\delta W=e c \int E_{\|} d t \approx-\frac{e c}{2 \pi R_{0}} \int L \frac{d I p}{d t} d t \approx \frac{e c L}{2 \pi R_{0}} \delta I_{p}
$$

$\left(\delta I_{p} \equiv I_{p}-I_{r} ; L \approx \mu_{0} R_{0}\right)$. It gives an energy range of 3-17 MeV for our discharges, consistent with the maximum energy measured by the $\gamma$-ray system when it did not saturate during the disruption. As the total energy of the runaway beam is $W \approx N_{r} \delta W$ and the number of runaway electrons $N_{r} \propto I_{r}, W$ scales as $W \propto I_{r}\left(I_{p}-I_{r}\right)$, reaching its maximum when the runaway current is $\sim 50 \%$ of the predisruptive plasma current. The most interesting experimental observation is that the runaway current may reach up to $\sim 80 \%$ of the predisruptive current, much larger than what is observed even in the largest tokamaks. The largest fraction reported in JET is $\sim 50 \%$ [8].

Another remarkable fact is that, in these discharges, the largest runaway current plateaus correspond to the slowest current quench rates. In Fig. 3, the fraction of runaway current formed during the disruption is plotted against the

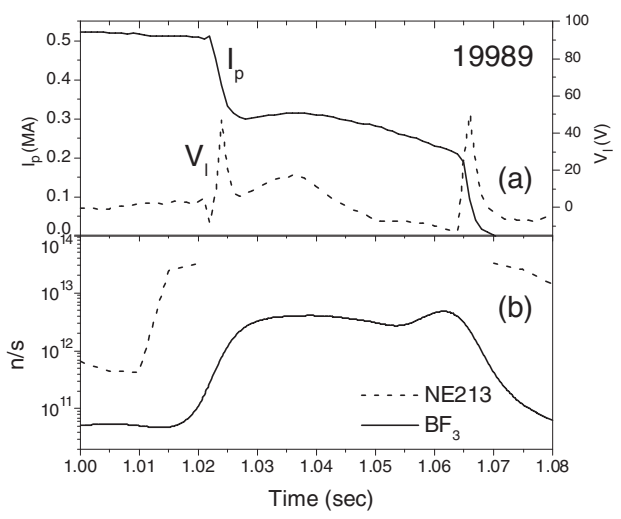

FIG. 2. Plasma disruption showing the formation of a $0.3 \mathrm{MA}$ runaway current: (a) Plasma current (solid line) and loop voltage (dashed line) traces; (b) neutron measurements: $B F_{3}$ (solid line) and NE213 (dashed line) signals. The absence of the NE213 line during the plateau phase is due to saturation. maximum plasma current quench rate. This fraction clearly decreases with the current derivative, which is in contrast to the increase observed in other devices [8]. The $I_{r} / I_{p}$ fraction expected from the Dreicer and avalanche mechanisms can be estimated by modeling the evolution of the runaway current profile $j_{r}$ self-consistently with the toroidal electric field $E_{\|}$obtained from the induction equation in cylindrical geometry,

$$
\mu_{0} \frac{\partial j_{p}}{\partial t}=\frac{1}{r} \frac{\partial}{\partial r}\left[r \frac{\partial E_{\|}}{\partial r}\right]=\frac{1}{r} \frac{\partial}{\partial r}\left[r \frac{\partial \eta\left(j_{p}-j_{r}\right)}{\partial r}\right] .
$$

$\eta$ is the resistivity, and we assume that the plasma current $j_{p}$ is replaced by the runaway current during the current decay $[1,6]$. The latter is estimated by assuming that all runaway electrons travel at the speed of light $\left(j_{r}=e n_{r} c\right)$ and considering primary and secondary generation:

$$
\frac{d n_{r}}{d t}=n_{e} \nu_{\mathrm{coll}} \lambda+\frac{n_{r}}{\tau_{s}},
$$

where $\nu_{\text {coll }}$ is the collision frequency, $\lambda$ is the (relativistic) Dreicer birth rate factor [17], and $\tau_{s}$ is the characteristic avalanching time $[7,18]$. Again, the result [shown in Fig. 3 with dash-dotted lines for two values of the density during the current decay: $n_{e}=5 \times 10^{19} \mathrm{~m}^{-3}$ (lower) and $n_{e}=$ $2 \times 10^{19} \mathrm{~m}^{-3}$ (upper)] is that $I_{r} / I_{p}$ should increase with the quenching rate, which is opposite to the trend we observe in these experiments. Not only that, but the resulting runaway current is too low as well. In fact, one would need to assume a disruption electron density of $\sim 2 \times$ $10^{19} \mathrm{~m}^{-3}$ to reproduce the observed runaway current fractions $\sim 50 \%$. This value is, however, quite a bit lower than the predisruption electron density, specially since one expects the density to increase during the disruption due to impurity influx (as observed in FTU).

The explanation we propose for the large runaway production observed in these discharges is that the predisruptive suprathermal electrons created during the LH injection phase (and detected by the FEB diagnostics) are accelerated during the disruption. This is very similar to the

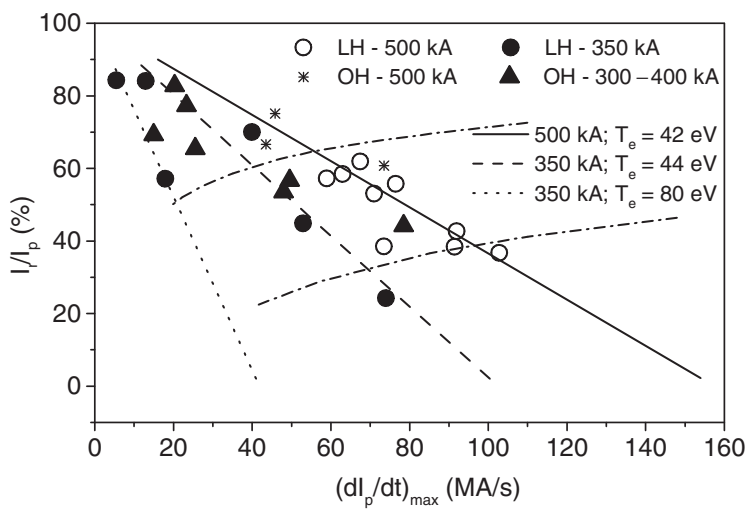

FIG. 3. Runaway current fraction vs maximum current derivative during current quench for $\mathrm{LH}$ runaway plateau disruptions. $\mathrm{OH}$ runaway plateau disruptions included for comparison. 
postheating situation already encountered at the end of nondisruptive LH discharges. The idea is that part of the suprathermal current survives during a disruptive event and becomes, at the end of the current quench, the observed runaway current plateau $I_{r}$. In this case, one would expect to find that $I_{r} \sim I_{\text {sth }}$ at the end of the current decay, $I_{\text {sth }}$ being the predisruption suprathermal current. Hence, the resistive part of the predisruption current, $I_{\text {res }}=I_{p}-I_{\text {sth }}$, would be quenched by the disruption, the nonresistive part, $I_{\text {sth }}$, constituting the final current plateau. Large runaway current plateaus would thus be related to small $I_{\text {res }}$ and therefore to small values of the electric field $\left(E_{\|}=\eta j_{\text {res }}\right.$; $\left.j_{\text {res }}=j_{p}-j_{r}\right)$ and current derivative $\left(d I_{p} / d t\right)$. This is indeed what is observed in the experiment as Fig. 3 shows. In fact, numerical simulations of the current profile evolution [using Eqs. (2) and (3)] that assume an initial resistive current $I_{\text {res }}=\left(I_{p}-I_{r}\right)$ reproduce fairly well the current evolution observed in all the cases using electron postthermal-quench temperatures $T_{e} \sim 40-50 \mathrm{eV}$ and a sufficiently large density (typically $\sim 10^{20} \mathrm{~m}^{-3}$ ) to avoid significant thermal electron runaway generation via primary or secondary mechanisms. A linear dependence between $I_{r} / I_{p}$ and $\left(d I_{p} / d t\right)_{\max }$ is also obtained that fits the experimental data for $T_{e}=40-45 \mathrm{eV}$. [In Fig. 3, calculations are shown for predisruptive plasma current values: $I_{p}=$ $500 \mathrm{kA}$ (solid line) and $I_{p}=350 \mathrm{kA}$ (dashed line).] Only in a few cases is the current evolution so slow that a larger $T_{e} \sim 80-100 \mathrm{eV}$ (dotted line) must be assumed.

There is a second way to confirm that these runaway electrons are formed via the acceleration of LH suprathermal electrons. If $\left(d I_{p} / d t\right)_{\max }$ (to a great extent determined by the resistive current at the start of the disruption) is plotted vs $I_{p}-I_{r}$ for all discharges with similar electron temperatures, all the data should collapse onto the same straight line independently of the predisruptive current $I_{p}$. This is indeed observed as shown in Fig. 4 (the straight line is obtained assuming $T_{e}=42 \mathrm{eV}$, the value that best fits the experimental data). Moreover, if the data of those FTU Ohmic $(\mathrm{OH})$ disruptions in which a runaway current plateau is formed are included, they are consistent with the trend exhibited by the LH cases (see Figs. 3 and 4). Since these disruptions occur mostly during the plasma current ramp-up or in low density discharges in which a large runaway population is detected prior to the disruption, this suggests that the acceleration of preexisting suprathermal electrons during the current quench is also responsible for the formation of their runaway current plateau.

In summary, large runaway currents (up to $\sim 80 \%$ of the predisruption plasma current) have been observed during disruptions occurring within the flattop phase of LH heated FTU discharges. In contrast to the theoretical expectations for electron thermal runaway generation (based on the usual Dreicer and avalanche mechanisms) and data from other tokamak experiments, the largest runaway currents

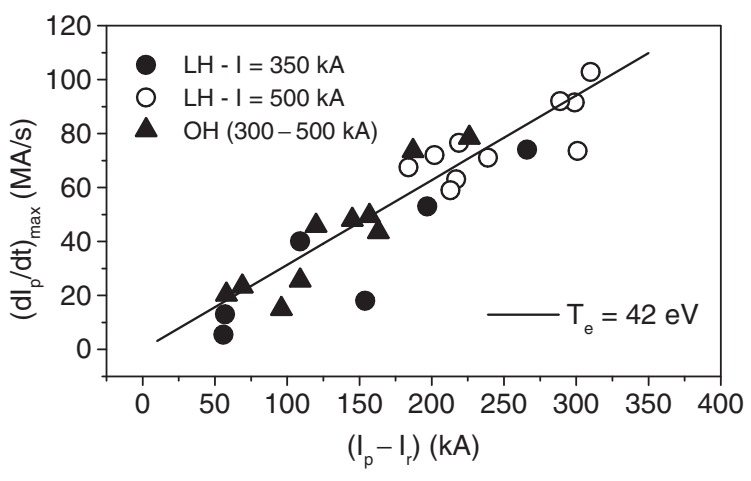

FIG. 4. Maximum current derivative during the disruption current quench vs $I_{p}-I_{r}$ for the LH runaway plateau disruptions. Ohmic runaway plateau disruptions included for comparison.

correspond to the slowest current decay rates. Acceleration of preexisting suprathermal electrons resonant with the LH waves during the disruption current quench explains the formation of such runaway currents. The same mechanism is also acting in FTU OH disruptions with runaway current plateau (in which a predisruption fast electron population is present).

Research supported in part by Spanish DGES Project No. FTN2003-04587. This work was also supported by the Euratom Communities under the contract of association between EURATOM and ENEA. The authors also thank Dr. P. Buratti for useful comments.

*Corresponding author.

Electronic address: solis@ fis.uc3m.es

[1] R. Jaspers et al., Nucl. Fusion 36, 367 (1996).

[2] J. A. Wesson et al., Nucl. Fusion 29, 641 (1989).

[3] R. Yoshino, J. Nucl. Mater. 220-222, 132 (1995).

[4] H. Dreicer, Phys. Rev. 117, 329 (1960).

[5] R. Jayakumar et al., Phys. Lett. A 172, 447 (1993).

[6] L.-G. Eriksson et al., Phys. Rev. Lett. 92, 205004 (2004).

[7] M. N. Rosenbluth et al., Nucl. Fusion 37, 1355 (1997).

[8] V. Plyusnin et al., Nucl. Fusion 46, 277 (2006).

[9] R. Andreani et al., in Fusion Technology (North-Holland, Amsterdam, 1990), Vol. 1, p. 218.

[10] G. Maddaluno et al., J. Nucl. Mater. 266-269, 593 (1999).

[11] J. R. Martin-Solis et al., Nucl. Fusion 45, 1524 (2005).

[12] B. Esposito et al., Phys. Plasmas 10, 2350 (2003).

[13] L. Bertalot et al., Rev. Sci. Instrum. 63, 4554 (1992).

[14] B. Esposito et al., Nucl. Instrum. Methods Phys. Res., Sect. A 476, 522 (2002).

[15] J. R. Martin-Solis et al., Nucl. Fusion 44, 974 (2004).

[16] J. A. Wesson et al., Nucl. Fusion 30, 1011 (1990).

[17] J. W. Connor et al., Nucl. Fusion 15, 415 (1975).

[18] S. Putvinski et al., Plasma Phys. Controlled Fusion 39, B157 (1997). 